\title{
RATE-DEPENDENT INVERSE HYSTERESIS FEEDFORWARD CONTROLLER FOR MICROSURGICAL TOOL
}

\author{
F. Alija Garmón, W. T. Ang, P. K. Khosla, and C. N. Riviere \\ The Robotics Institute, Carnegie Mellon University, Pittsburgh, PA 15213, USA
}

\begin{abstract}
This paper presents the development and initial results of a controller based on a novel rate-dependent hysteresis model. The controller has been developed for "Micron," a microsurgical instrument designed to sense and actively cancel tremor and other undesired motion during vitreoretinal intervention. To accomplish this, Micron uses a three-degree-of-freedom parallel manipulator with piezoelectric actuation. In order to achieve high accuracy it is necessary to deal with the hysteresis of the piezoelectric actuators. Most hysteresis models are based on elementary rate-independent operators and are not suitable for modeling actuator behavior across a wide frequency band. This work proposes a rate-dependent controller, based on a modification of the rate-independent Prandtl-Ishlinskii (PI) model, to counteract hysteresis during open-loop control of piezoelectric actuators tracking multi-frequency motion profiles. In experiments tracking real tremor, it consistently outperforms its rate-independent counterpart.
\end{abstract} tremor

Keywords-Hysteresis, piezoelectric, rate-dependence,

\section{INTRODUCTION}

Humans have innate limitations in manual positioning accuracy. This phenomenon hinders accuracy in micromanipulation, including microsurgical procedures [1].

Efforts to attain high precision have included the use of telerobotic technology [2], where the unstable human arm is replaced by a robotic arm; the "steady-hand" robotic system [3], where the tool is hold simultaneously both by the operator's hand and an actively controlled robot arm; and a fully hand-held active instrument, developed in our laboratory and known as "Micron" [4], which senses and actively compensates unwanted movement.

The most familiar type of erroneous movement affecting microsurgery is tremor, defined as any involuntary and roughly sinusoidal movement [5]. Physiological tremor is a type of tremor that is inherent in the movement of healthy subjects such as surgeons. In ophthalmological microsurgery, the most significant component of the physiological tremor is found to be an oscillation at $8-12 \mathrm{~Hz}$ [6] and up to $50 \mu \mathrm{m}$ peak-to-peak (p-p) vector magnitude [7]. The compensation therefore must at least cover this frequency band. For tremor canceling, piezoelectric actuators were chosen due to their miniature size, high output force, high bandwidth and fast response. However, the existence of nonlinear rate-dependent multi-path hysteresis in piezoelectric material produces maximum errors of about $15 \%$ in static positioning applications and growing with the rate. Since the manipulator system of
Micron is intended to yield $100 \mu \mathrm{m}$ total travel in each principal axis with $1 \mu \mathrm{m}$ rms noise, hysteretic nonlinearity must be eliminated.

In order to avoid hysteretic effects, three primary options exist: closed-loop displacement control, charge control, and open-loop control using an inverse feedforward controller. For this application, open-loop control has been chosen for its simplicity as well as for its guaranteed stability. The main idea of open-loop feedforward inverse control is to construct a mathematical model that closely describes the complex hysteretic behavior, to obtain the inverse of this model and use this inverse model as a controller in cascade with the real actuator in order to linearize its response (Fig. 1). The accuracy of the model determines the accuracy of the control system. The main drawback of the inverse feedforward controller is therefore the possibility of unmodeled external disturbances, e.g., temperature variation. In such a case, the feedforward model can be combined with feedback control to optimize both static and dynamic performance.

The hysteresis model used is based on the rateindependent Prandtl-Ishlinskii (PI) operator [8, 9], due to its suitability for real time applications and the existence of a closed form solution for its inverse. This paper presents a particular treatment of PI-operator-based modeling to account for the non-negative input voltage requirement of the piezoelectric actuators employed, and then extends the model to include rate-dependent characteristics of the piezoelectric hysteresis. We have shown experimentally that the relationship between the slope of the hysteretic loading curve and the rate of control input can be modeled by a linear function.

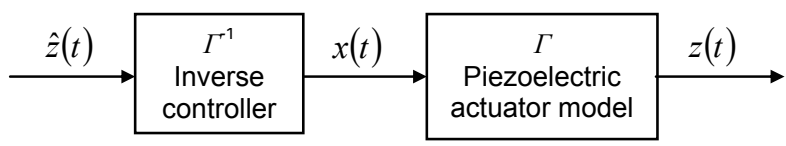

Fig. 1. Piezoelectric actuator linearization with feedforward inverse hysteresis controller. The input of the controller is the desired displacement and output $x(t)$ is the voltage to apply to the piezoelectric actuator after amplification.

\section{RATE-INDEPENDENT PI HYSTERESIS MODEL}

\section{A. Hysteresis Operator}

The elementary operator in the PI hysteresis model is a rate-independent backlash or linear-play operator defined by 


$$
y(t)=H_{r}\left[x, y_{0}\right](t)=\max \{x(t)-r, \min \{x(t)+r, y(t-T)\}\}
$$

where $x$ is the input, $y$ is the output, $r$ is the input threshold value or the magnitude of the backlash, and $T$ is the sampling period. Multiplying the backlash operator $H_{r}$ by a weight value $w_{h}$, the generalized backlash operator is

$$
y(t)=w_{h} \cdot H_{r}\left[x, y_{0}\right](t) \text {. }
$$

The weight $w_{h}$ defines the gain of the backlash operator $\left(w_{h}=1\right.$ represents a $45^{\circ}$ slope $)$ and may be viewed as the gear ratio in a mechanical gear play analogy (see Fig. 2).

Complex hysteretic nonlinearity can be modeled by a linearly weighted superposition of many backlash operators with different threshold and weight values,

$$
y(t)=\boldsymbol{w}_{\boldsymbol{h}}{ }^{\mathrm{T}} \cdot \boldsymbol{H}_{\boldsymbol{r}}\left[x, \boldsymbol{y}_{\mathbf{0}}\right](t)
$$

with the weight vector $\boldsymbol{w}_{\boldsymbol{h}}{ }^{\mathrm{T}}=\left[\begin{array}{llll}w_{h 0} & \ldots & w_{h n}\end{array}\right]$, the operators $\boldsymbol{H}_{\boldsymbol{r}}\left[x, \boldsymbol{y}_{0}\right](t)=\left[H_{r 0}\left[x, y_{00}\right](t) \ldots H_{r n}\left[x, y_{0 n}\right](t)\right]^{\mathrm{T}}$, the threshold vector $\boldsymbol{r}=\left[\begin{array}{lll}r_{0} & \ldots & r_{n}\end{array}\right]^{\mathrm{T}}$ (where $\left.0=r_{0}<\ldots<r_{n}\right)$, and the initial state vector $\boldsymbol{y}_{\mathbf{0}}=\left[\begin{array}{lll}y_{00} & \ldots & y_{0 n}\end{array}\right]^{\mathrm{T}}$. The threshold parameters, $\boldsymbol{r}$, are distributed from 0 to midrange according to

$$
r_{i}=\sum_{j=0}^{i} j, i=0 \ldots n .
$$

Using this arrangement, the growing distance between consecutive $r$ 's results in a larger concentration of backlash operators at the turning points of the control input signal. This increases the accuracy of the model, since the turning points are the most important part of the modeling. The distribution only to midrange is due to the one-sided characteristic of the piezoelectric actuators.

\section{B. Saturation Operator}

A PI operator formed with backlash operators alone cannot model two common hysteretic phenomena: asymmetrical shape and saturation at high input levels. To overcome this restrictive property, a saturation operator is combined in series with the hysteresis operator. A saturation operator is a weighted linear superposition of one-sided dead-zone operators. A dead zone is a nonlinear operator given by:

$$
\begin{aligned}
& S_{d}[y](t)= \begin{cases}\max \{y(t)-d, 0\}, & d>0 \\
y(t), & d=0\end{cases} \\
& z(t)=\boldsymbol{w}_{\boldsymbol{s}}^{\mathrm{T}} \cdot S_{d}[y](t)
\end{aligned}
$$

where $y$ is the output of the hysteresis operator, $z$ is the actuator response, $\boldsymbol{w}_{s}{ }^{\mathrm{T}}=\left[\begin{array}{lll}w_{s 0} & \ldots & w_{s m}\end{array}\right]$ is the weight vector, and $\boldsymbol{S}_{d}[x](t)=\left[S_{d 0}[x](t) \ldots S_{d m}[x](t)\right]^{\mathrm{T}}$ with the threshold vector $\boldsymbol{d}=\left[\begin{array}{lll}d_{0} & \ldots & d_{m}\end{array}\right]^{\mathrm{T}}$ (where $\left.0=d_{0}<r_{n}<d_{1}<\ldots<d_{m}\right)$. The reason to distribute $d_{1}$ to $d_{\mathrm{m}}$ over the midrange point is that saturation affects only high values of the control input.

Placing the hysteresis and saturation operators yields the rate-independent PI operator

$$
z(t)=\Gamma[x](t)=\boldsymbol{w}_{\boldsymbol{s}}^{\mathrm{T}} \cdot \boldsymbol{S}_{d}\left[\boldsymbol{w}_{\boldsymbol{h}}{ }^{\mathrm{T}} \cdot \boldsymbol{H}_{r}\left[x, \boldsymbol{y}_{0}\right]\right](t) .
$$

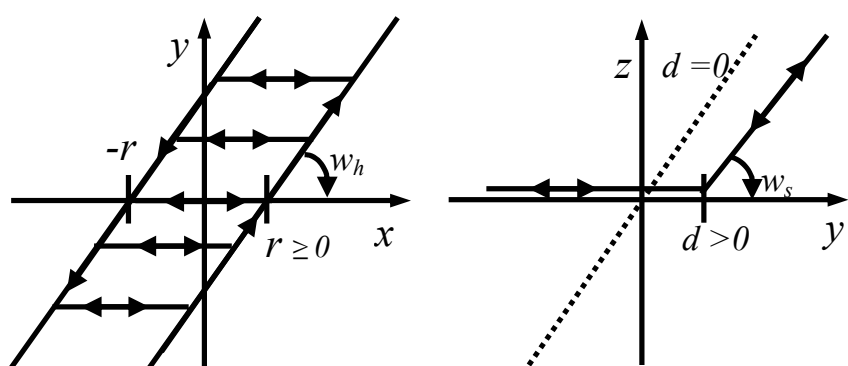

Fig. 2. The rate-independent generalized backlash operator (at left) is characterized by the threshold, $r$, and the weight, $w_{h}$. The one-sided deadzone operator (at right) is characterized by the threshold, $d$, and the gain $w_{s}$

\section{Rate-Independent Inverse PI Model}

The inverse represents the reflection of the hysteretic loop about the $45^{\circ}$ line. The proof of existence of an inverse can be found in [10]. The inverse PI operator is given by

$$
\Gamma^{-1}[z](t)=\boldsymbol{w}_{\boldsymbol{h}}{ }^{\mathrm{T}} \cdot \boldsymbol{H}_{\boldsymbol{r}},\left[\boldsymbol{w}_{\boldsymbol{s}}{ }^{\mathrm{T}} \cdot \boldsymbol{S}_{\boldsymbol{d}},[z], \boldsymbol{y}_{\mathbf{0}}{ }^{\mathrm{T}}\right](t)
$$

and can be calculated analytically using the parameter transformation laws presented in [10]. Cascading the inverse hysteresis model with the actual hysteresis model, as in Fig. 1, leads of course to the identity operator between the control input $\hat{z}(t)$ and actuator response $z(t)$ :

$$
z(t)=\Gamma\left[\Gamma^{-1}[\hat{z}]\right](t)=I[\hat{z}](t)=\hat{z}(t) .
$$

\section{Parameter Identification}

To find the hysteresis model parameters, the responses of the actuator first have to be measured. Then, the threshold values $\boldsymbol{r}$ and $\boldsymbol{d}$ are set as described in the previous section, and finally weight parameters $\boldsymbol{w}_{h}$ and $\boldsymbol{w}_{s}$ are found by performing a least squares minimization of the error

$$
E[x, z]\left(\boldsymbol{w}_{\boldsymbol{s}}, \boldsymbol{w}_{\boldsymbol{h}}, t\right)=\boldsymbol{w}_{\boldsymbol{s}}^{\mathrm{T}} \cdot \boldsymbol{S}_{\boldsymbol{d}}\left[\boldsymbol{w}_{\boldsymbol{h}}^{\mathrm{T}} \cdot \boldsymbol{H}_{\boldsymbol{r}}\left[x, \boldsymbol{y}_{\mathbf{0}}\right]\right](t)-z(t) .
$$

\section{RATE-DEPENDENT PI HYSTERESIS MODEL}

An extension to the rate-independent PI model is proposed in this section to also model the rate-dependent characteristics of the piezoelectric hysteresis.

1) Rate-dependent Model Identification: The response of a piezoelectric actuator is measured tracking constant-rate sawtooth control inputs at different rate values over the range of $0-1000 \mu \mathrm{m} / \mathrm{s}$. This corresponds to the maximum rate of sinusoidal control input up to $25 \mathrm{~Hz}$ with $12.5 \mu \mathrm{m}$ p$\mathrm{p}$ amplitude. PI parameter identification is performed for the measured actuator response at different rate values. The sums of the hysteresis operator weights, defined as

$$
W_{h i}=\sum_{j=0}^{i} w_{h j}, i=0 \ldots n,
$$

are plotted against control input rate in Fig. 3. 


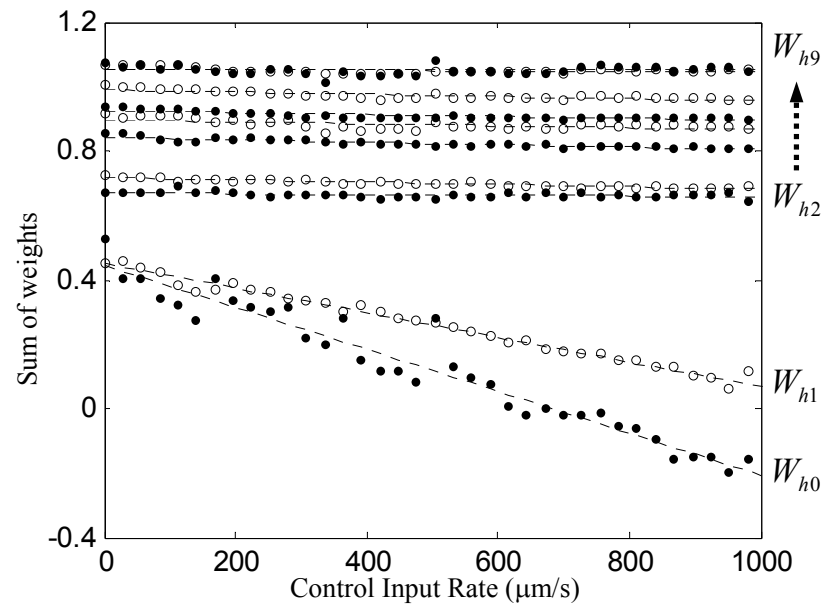

Fig.3. Plot of the sum of the hysteresis operator weights vs. input rate. Linear dependence can be observed.

The sum of the weights is used instead of one or more individual weight values because the linear fit is more accurate. A standard deviation of 0.04 is the result for $W_{h 0}$, making it the roughest linear fit among all $W_{h i}$.

Thus the rate-dependent hysteresis slope model is:

$$
W_{h i}(\dot{x}(t))=A_{i} \cdot \dot{x}(t)+B_{i}, i=0 \ldots n
$$

where $A_{i}$ is the slope of the best fit line and $B_{i}$ is the hysteresis slope at zero input rate. The individual ratedependent weight values can be calculated by

$$
w_{h i}(\dot{x}(t))=a_{i} \cdot \dot{x}(t)+b_{i}, i=0 \ldots n
$$

where

$$
\begin{aligned}
& a_{0}=A_{0} ; a_{i}=A_{i}-A_{(i-1)}, i=1 \ldots n \\
& b_{0}=B_{0} ; b_{i}=B_{i}-B_{(i-1)}, i=1 \ldots n .
\end{aligned}
$$

2) Rate-dependent PI Hysteresis Model: With the ratedependent extension, the PI hysteresis model is defined by

$$
z(t)=\Gamma[x, \dot{x}](t)=\boldsymbol{w}_{\boldsymbol{s}}{ }^{\mathrm{T}} \cdot \boldsymbol{S}_{\boldsymbol{d}}\left[\boldsymbol{w}_{\boldsymbol{h}}(\dot{x}(t))^{\mathrm{T}} \cdot \boldsymbol{H}_{\boldsymbol{r}}\left[x, \boldsymbol{y}_{\mathbf{0}}\right]\right](t) .
$$

The inverse rate-dependent modified PI operator is as follows:

$$
\left.\Gamma^{-1}[\hat{z}](t)=\boldsymbol{w}_{\boldsymbol{h}}^{\prime}(\dot{x}(t))^{\mathrm{T}} \cdot \boldsymbol{H}_{\boldsymbol{r}^{\prime}},(\dot{x}(t))\left[\boldsymbol{w}_{\boldsymbol{s}}^{, \mathrm{T}} \cdot \boldsymbol{S}_{d^{\prime}}, \hat{z}\right], \boldsymbol{y}_{\mathbf{0}} \mathbf{}\right](t) .
$$

The rate-dependent transformation laws for $\boldsymbol{w}_{\boldsymbol{h}}{ }_{\boldsymbol{h}}(\dot{x}(t))$ can be found in the same way as the rate-independent, by replacing $\boldsymbol{w}_{\boldsymbol{h}}$ with the rate-dependent $\boldsymbol{w}_{\boldsymbol{h}}(\dot{x}(t))$.

\section{RESUlts}

To model the hysteretic nonlinearity of a TS18-H5-104 piezoelectric stack actuator (Piezo Systems, Inc., Cambridge, Mass.), we used a PI model of $n=9$ and $m=2$, for both the rate-independent and rate-dependent cases. The rate-independent model parameters were identified from the measured response of the piezoelectric actuator subjected to a $10 \mathrm{~Hz}, 12.5 \mu \mathrm{m}$ p-p sinusoidal control input.

Fig. 4 compares the experimental results of the rateindependent and rate-dependent PI operators tracking real tremor. Recordings of the hand motion of ophthalmologic surgeons [11] were made available by Prof. R. S. Rader of The Johns Hopkins University. Each surgeon held a microsurgical instrument with the tip inserted in a sclerotomy in the eye of a mannequin face. A Hall effect sensor mounted inside the mannequin eye detected the position, in one dimension, of a $0.26 \mathrm{~g}$ permanent magnet mounted on the tip in the instrument. Data were recorded for 16 seconds and at $1 \mathrm{kHz}$ sampling frequency. The tremor was then estimated using a bandpass filter. The corner frequencies were set at 6 and $16 \mathrm{~Hz}$ to provide flat response in the $8-12 \mathrm{~Hz}$ band. Table I summarizes the performance of the rate-independent and rate-dependent controllers during the experiment.

TABLE I

MEASURED EXPERIMENTAL ERRORS DURING TRACKING OF REAL TREMOR

\begin{tabular}{rccc}
\hline & \multicolumn{3}{c}{ Results Comparison } \\
\cline { 2 - 4 } & Raw hysteresis & Rate-Independent & Rate-Dependent \\
\hline $\begin{array}{r}\text { Rmse }(\mu \mathrm{m}) \\
\text { Rmse / p-p } \\
\text { ampl. }(\%)\end{array}$ & 0.86 & 0.29 & 0.08 \\
$\begin{array}{r}\text { max error } \\
(\mu \mathrm{m})\end{array}$ & 1.82 & 2.32 & 0.64 \\
$\begin{array}{r}\text { max error / p- } \\
\text { p ampl. }(\%)\end{array}$ & 14.5 & 0.66 & 0.32 \\
\hline
\end{tabular}

\section{DISCUSSION}

In order to suppress erroneous motion, the actuators of Micron must move the tool tip in opposition to the tremor; i.e., they should track a tremulous motion. Due to the modulating or nonstationary nature of the tremor, rate effects must be taken into account. The rate-independent controller, though it reduces significantly the hysteretic effects of the piezoelectric actuators, is outperformed by the rate-dependent controller. The rate-dependent open-loop controller permits attainment of the goal of $0.125 \mu \mathrm{m} \mathrm{rms}$ error per actuator, as specified in the design of Micron.

One limitation of all PI-type hysteresis models is singularity when the slope of the hysteresis loading curve becomes zero. The inverse model near the singularity is highly sensitive to noise, wherefore the range of dependence must be bounded to avoid control errors. The singularity of the proposed rate-dependent model occurs at around 700 $\mu \mathrm{m} / \mathrm{s}$, which is equivalent to the maximum rate of a $20 \mathrm{~Hz}$, $12.5 \mu \mathrm{m}$ p-p sinusoid. However, Micron actuators track motions only up to approximately $12 \mathrm{~Hz}$, therefore they work in the safe range. If desired, wider spacing of weights could be used for higher bandwidth. 


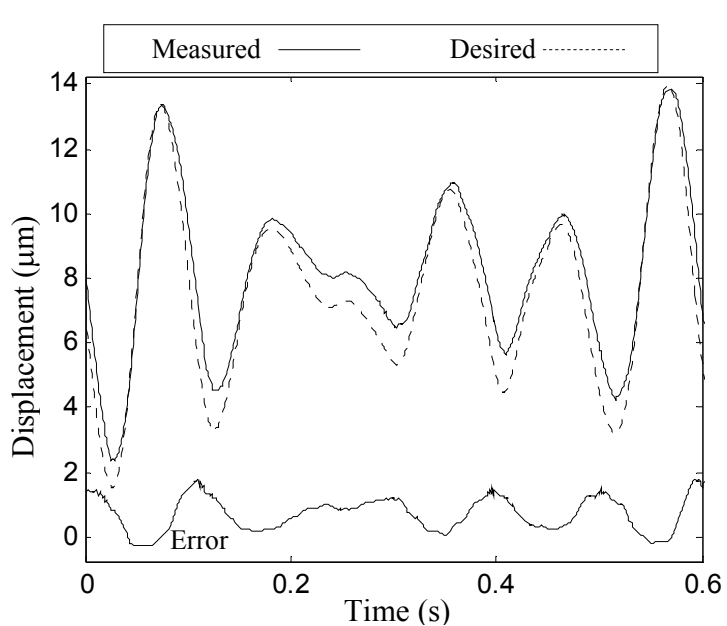

(a)

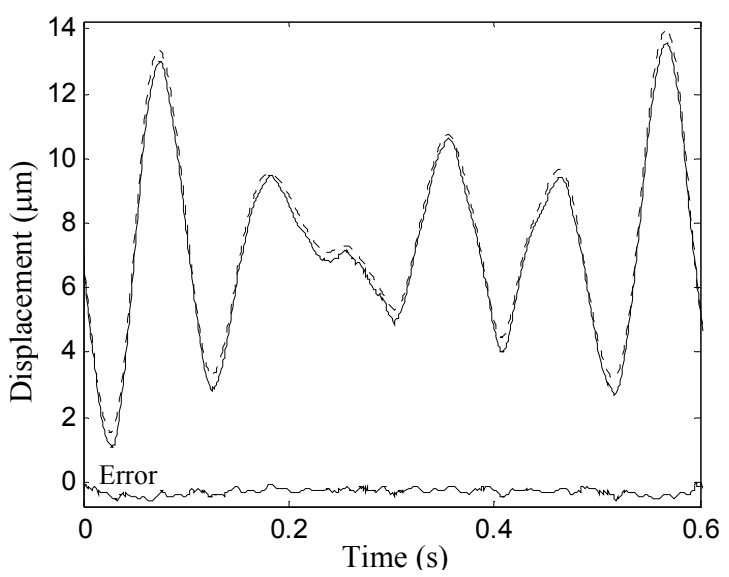

(b)

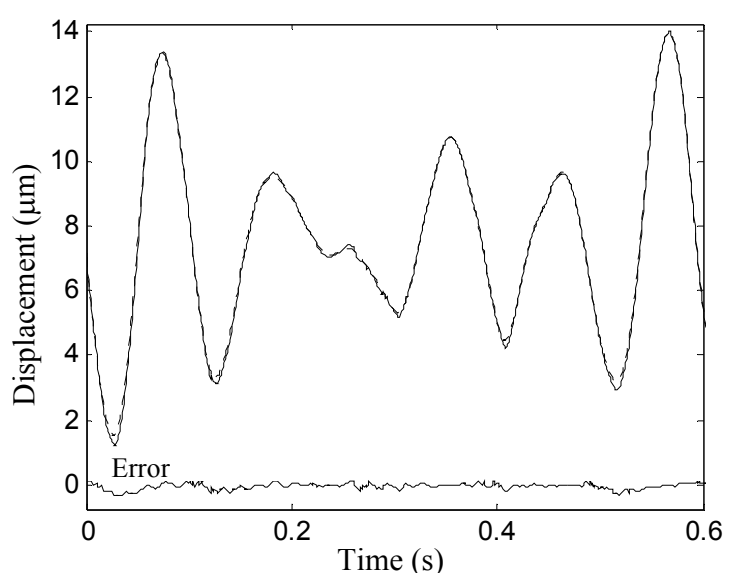

(c)

Fig.9. Experimental open-loop tracking results for real tremor. The rate-independent model parameters are identified from the measured response of the piezoelectric actuator subjected to a 10 $\mathrm{Hz}, 12.5 \mu \mathrm{m}$ p-p sinusoidal input. (a) Without model. (b) Rateindependent hysteresis model. (c) Rate-dependent model.

\section{CONCLUSION}

A novel rate-dependent controller based on the PrandtlIshlinskii (PI) rate-independent hysteresis model has been presented, to account for the behavior of a piezoelectric actuator in an active tremor-canceling microsurgical instrument. The proposed system uses a linear function to model the relationship between the parameters of the hysteresis operators and the rate of control input. This controller yields better experimental results than the rateindependent controller.

\section{REFERENCES}

[1] R. C. Harwell and R. L. Ferguson, "Physiologic tremor and microsurgery," Microsurgery, vol. 4, pp. 187-192, 1983.

[2] I. W. Hunter, T. D. Doukoglou, S. R. Lafontaine, P. G. Charette, L. A. Jones, M. A. Sagar, G. D. Mallinson, and P. J. Hunter, "A teleoperated microsurgical robot and associated virtual environment for eye surgery," Presence, 2:265-280, 1993.

[3] R. Taylor, P. Jensen, L. Whitcomb, A. Barnes, R. Kumar, D. Stoianovici, P Gupta, Z. Wang, E. de Juan, and L. Kavoussi, "A steady-hand robotic system for microsurgical augmentation," in: C. Taylor, A. Colchester (eds.) Medical Image Computing and Computer-Assisted Intervention - MICCAI'99. Springer, Berlin, pp. 1031-1041.

[4] W. T. Ang, C. N. Riviere, and P. K. Khosla, "Design and implementation of active error canceling in a hand-held microsurgical instrument," Proc. IEEE/RSJ Int. Conf. Intell. Robots and Systems, Hawaii, Oct. 29-Nov. 3, 2001.

[5] R. J. Elble and W.C. Koller, Tremor. Baltimore: Johns Hopkins, 1990.

[6] C. N. Riviere, R. S. Rader, and P. K. Khosla, "Characteristics of hand motion of eye surgeons," Proc. $19^{\text {th }}$ Int. Conf. IEEE Eng. Med. Biol. Soc., Chicago, 1997.

[7] S. P. N. Singh, C. N. Riviere, "Physiological tremor amplitude during retinal microsurgery," Proc. 28th IEEE Northeast Bioeng. Conf., Phila. Pa., 2002, pp. 171-172.

[8] P. Krejci and K. Kuhnen, "Inverse control of systems with hysteresis and creep", IEE Proc. Control Theory Appl., vol. 148, no.3, May 2001.

[9] K. Kuhnen and H. Janocha, "Complex hysteresis modeling of a broad class of hysteretic nonlinearities", Proc. 8th Int. Conf. on New Actuators, Bremen, pp. 688-691, Jun. 2002.

[10] K. Kuhnen, H. Janocha, "Inverse feedforward control for the large signal operation of piezoelectric actuators"(in German), atAutomatisierungstechnik, vol. 50, 9/2002, S. 439-450.

[11] C. N. Riviere and P. K. Khosla, "Augmenting the humanmachine interface: Improving manual accuracy," Proc. IEEE Int. Conf. Robot. Autom., Albuquerque, N.M. Apr. 20-25, 1997. 